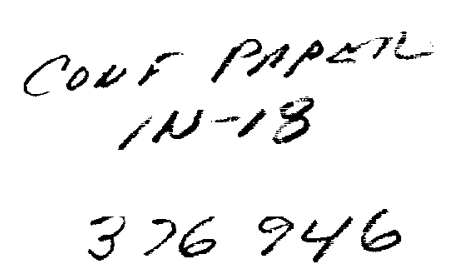

\title{
OVERVIEW OF FUTURE NASA TETHER APPLICATIONS
}

L. Johnson', B. Gilchrist ${ }^{2}$, R. D. Estes ${ }^{3}$, and E. Lorenzini ${ }^{3}$

'National Aeronautics and Space Administration, George C. Marshall Space Flight Center, Huntsville, Alabama, 35812, USA

${ }^{2}$ The University of Michigan Space Physics Research Laboratory, Ann Arbor, Michigan, 48109

${ }^{3}$ Smithsonian Astrophysical Observatory, Cambridge, Massachusetts, 02138

\section{ABSTRACT}

The groundwork has been laid for tether applications in space. NASA has developed tether technology for space applications since the 1960's. Important recent milestones include retrieval of a tether in space (TSS1, 1992), successful deployment of a 20-km-long tether in space (SEDS-1, 1993), and operation of an electrodynamic tether with tether current driven in both directions-power and thrust modes (PMG, 1993). Various types of tethers and systems can be used for space transportation. Short electrodynamic tethers can use solar power to 'push' against a planetary magnetic field to achieve propulsion without the expenditure of propellant. The planned Propulsive Small Expendable Deployer System (ProSEDS) experiment will demonstrate electrodynamic tether thrust during its flight in early 2000 . Utilizing completely different physical principles, long non-conducting tethers can exchange momentum between two masses in orbit to place one body into a higher orbit or a transfer orbit for lunar and planetary missions. Recently completed system studies of this concept indicate that it would be a relatively low-cost in-space asset with long-term multimission capability. Tethers can also be used to support space science by providing a mechanism for precision formation flying and for reaching regions of the upper atmosphere that were previously inaccessible.

\section{ELECTRODYNAMIC TETHER PROPULSION}

A predominantly uninsulated (bare wire) conducting tether, terminated at one end by a plasma contactor, can be used as an electromagnetic thruster. A propulsive force of $\mathbf{F}=\mathbf{I L} \times \mathbf{B}$ is generated on a spacecraft/tether system when a current, I, from an on-board power supply is fed into a tether of length, $\mathbf{L}$, against the emf induced in it by the geomagnetic field, $\mathbf{B}$. This concept will work near any planet with a magnetosphere (Earth, Jupiter, etc.) This was demonstrated by the Tethered Satellite System Reflight (TSS-1R) mission - the Orbiter experienced a $0.4 \mathrm{~N}$ electrodynamic drag thrust during tether operation.

An electrodynamic tether upper stage could be used as an orbital tug to move payloads within low earth orbit (LEO) after insertion (Figure 1). The tug would rendezvous with the payload and launch vehicle, dock/grapple the payload and maneuver it to a new orbital altitude or inclination within LEO without the use of boost propellant. The tug could then lower its orbit to rendezvous with the next payload and repeat the process. Such a system could conceivably perform several orbital maneuvering assignments without resupply, making it low recurring cost space asset. The performance of a $10 \mathrm{~kW}, 10 \mathrm{~km}$ tether system for altitude changes is illustrated in Figure 2. The same system can be used to change the orbital inclination of a payload as well. Figure 3 can be used to determine the available inclination change for a particular 
spacecraft and payload mass by dividing the 'specific inclination rate' indicated by the total system mass as a given altitude.

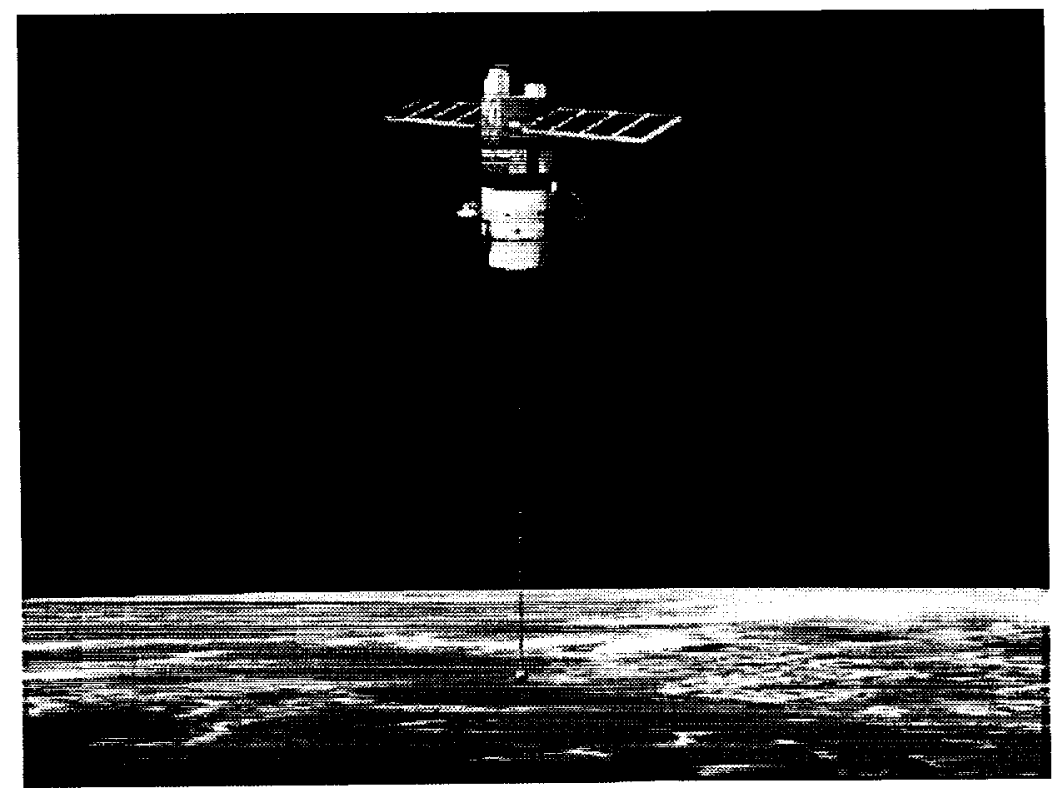

Fig. 1. Artist concept of the Electrodynamic Tether Upper Stage Demonstrator.

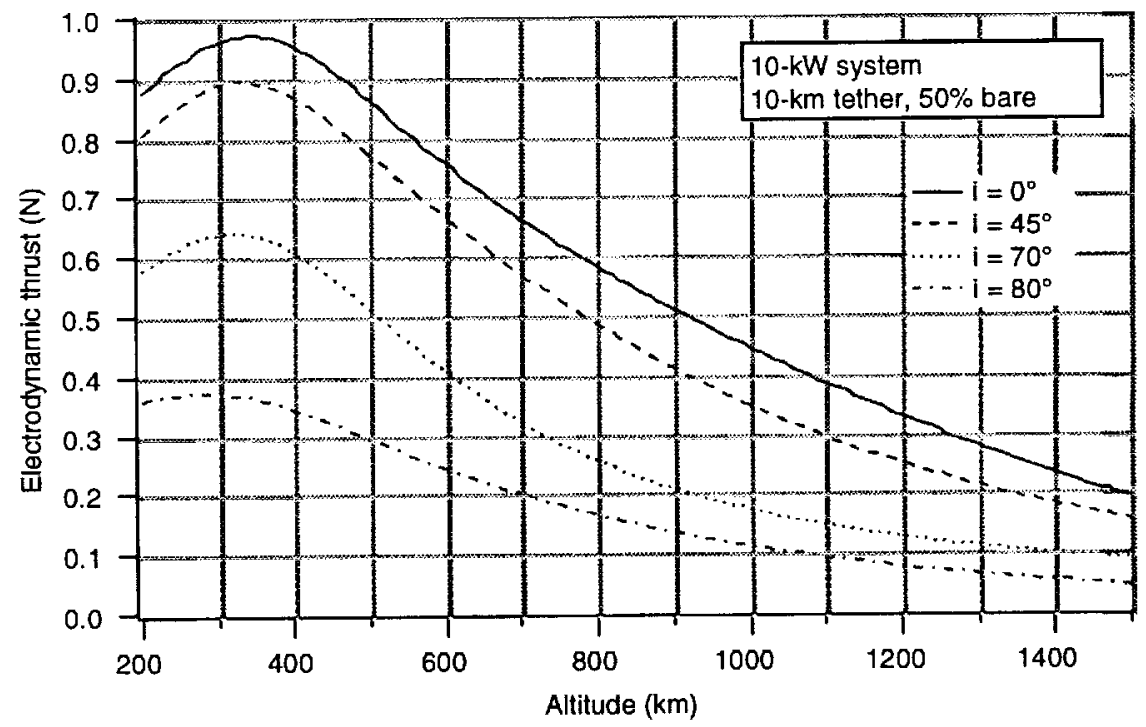

Fig. 2. The performance of an electrodynamic tether thruster varies with altitude in the ionosphere (where $\mathrm{i}$ is the orbital inclination). 


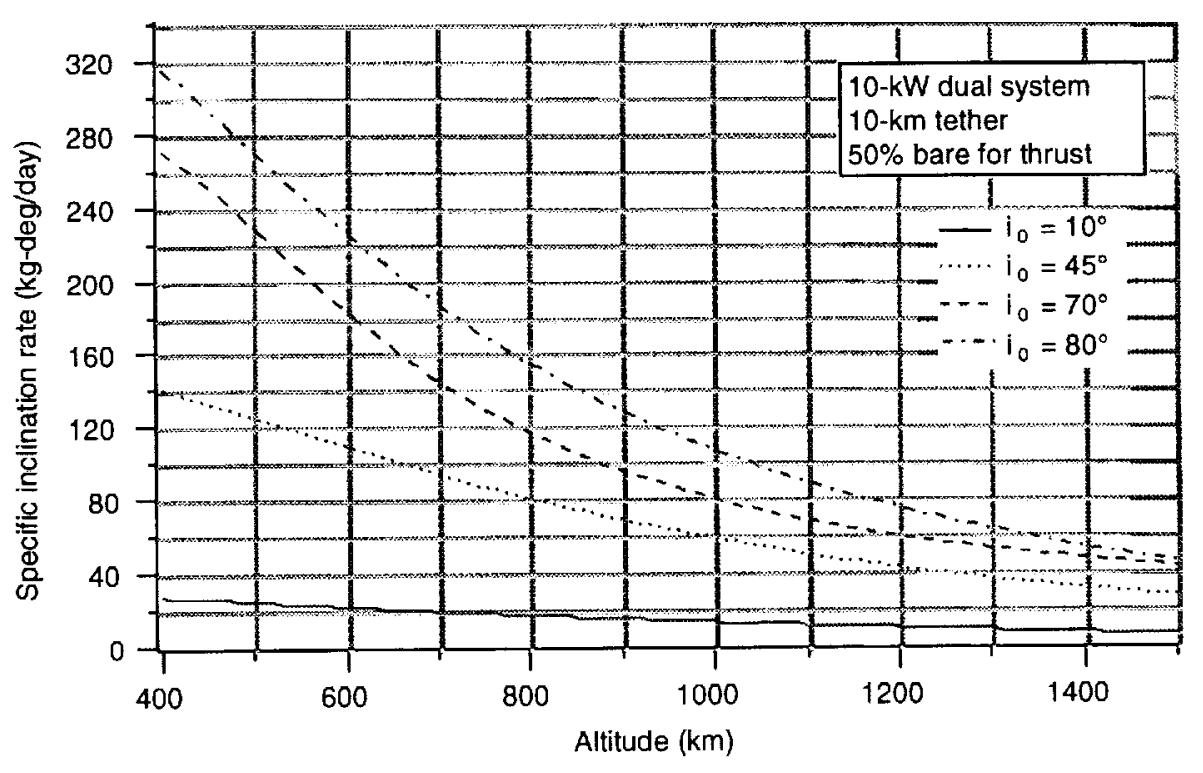

Fig. 3. The performance of an electrodynamic tethers thruster for inclination change applications depends strongly on the initial orbital inclination $\left(\mathrm{i}_{0}\right)$.

\section{ProSEDS Flight Experiment}

From theoretical analyses and preliminary plasma chamber tests, bare tethers appear to be very effective anodes for collecting electrons from the ionosphere and, consequently, attain high currents with relatively short tether lengths (Sanmartin and Martinez-Sanchez, 1993). A flight experiment to validate the performance of the bare electrodynamic tether in space and demonstrate its capability to produce thrust is planned by NASA for the year 2000. The ProSEDS (Propulsive Small Expendable Deployer System) experiment will be placed into a $400 \mathrm{~km}$ circular orbit as a secondary payload from a Delta II launch vehicle. The flight-proven SEDS will be used to deploy a $5 \mathrm{~km}$ predominantly bare aluminum wire attached to $20 \mathrm{~km}$ of insulating Spectra tether and $25 \mathrm{~kg}$ endmass.

Once on orbit, the deployer will reel-out the tether and endmass system to a total length of $25 \mathrm{~km}$. Upward deployment will set the system to operate in the generator mode, thus producing drag thrust and electrical power. The drag thrust provided by the tether will deorbit the Delta II upper stage in approximately three weeks, versus its nominal 1 year lifetime in a $400 \mathrm{~km}$ circular orbit. Approximately $100 \mathrm{~W}$ electrical power will be extracted from the tether to recharge mission batteries and to allow extended measurements of the system's performance until it reenters.

\section{'HANGING' MOMENTUM EXCHANGE TETHERS}

Momentum exchange tethers provide a unique advantage to reusable launch vehicle (RLV) systems for boosting payloads to higher orbits. This stems from the fact the RLV must reduce its altitude after payload release in order to return to Earth, while at the same time the payload it ejects must increase its altitude to reach the desired orbit. In other words, momentum must be removed from the RLV and added to the payload. Tethers provide a method to make this exchange (Colombo, 1981).

Operationally, this can be accomplished by the deployment of the payload upward on a long $(\sim 20 \mathrm{~km})$ tether from the RLV (Figure 4). Libration begins and the momentum is transferred from the heavy RLV to the payload. Upon release, the RLV experiences a deboosting force and the payload is inserted into an 
orbit with apogee many times the tether length higher than previously (Bekey, 1997). This was demonstrated inadvertently when the electrodynamic tether broke on the TSS-1R flight, sending the endmass into a new orbit. The technology for this type of momentum exchange utilizing a tether exists today and was successfully demonstrated with the SEDS in 1993 (Rupp and Carrington, 1993).

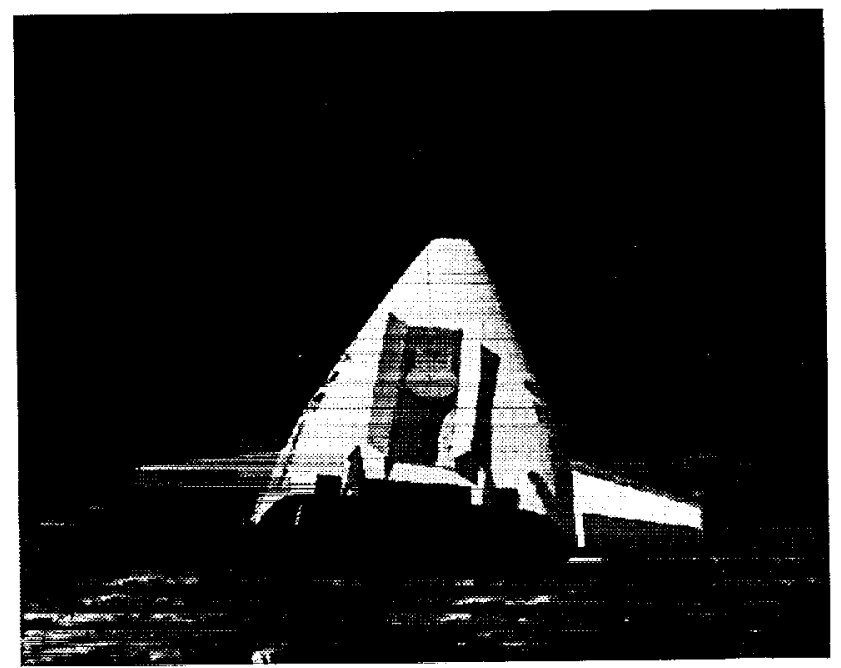

Fig. 4. Artist concept of an upper stage tether deployed from the Reusable Launch Vehicle.

The physics governing the momentum exchange is best illustrated in Figure 5. The tether system is stabilized along the local vertical by the gravity gradient force with the center of mass maintained at the orbital velocity for a given altitude. The satellite is thus at a superorbital velocity and the RLV at a suborbital one. After the tether is cut, the angular momentum is exchanges between the two masses: the center of mass of the orbit remains the same while the satellite is injected into a higher orbit with the perigee at the release location. The RLV is injected into a lower orbit with apogee at the release location.

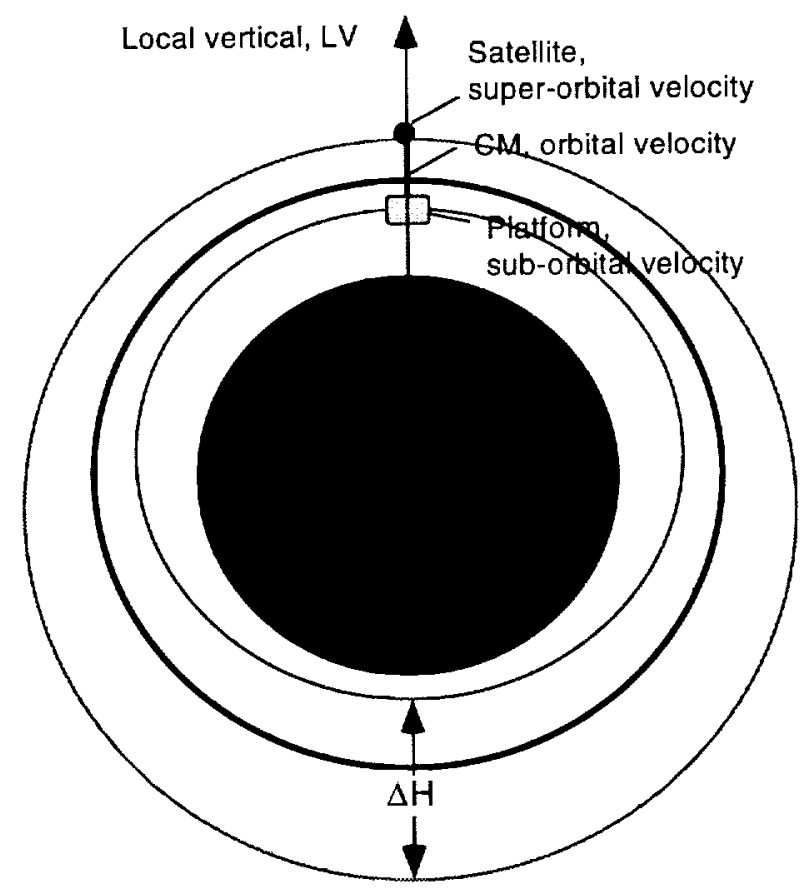

Fig. 5. Orbits of the launch vehicle and satellite after release in the 'hanging' tether boost scenario. 


\section{ROTATING MOMENTUM EXCHANGE TETHERS}

A spinning tether system can be used to boost payloads into higher orbits with a Hohmann-type transfer. A tether system would be anchored to a relatively large mass in LEO awaiting rendezvous with a payload delivered to orbit. The uplifted payload meets with the tether facility which then begins a slow spin-up using electrodynamic tethers (for propellantless operation) or another low thrust, high Isp thruster. At the proper moment and tether system orientation, the payload is released into a transfer orbit - potentially to geostationary transfer orbit (GTO) or Lunar Transfer Orbit (LTO). A network of such systems could be developed to "hand off" a payload until it reaches the desired location.

The physics governing a rotating momentum exchange system is illustrated in Figure 6. Following spin-up of the tether and satellite system, the payload is released at the local vertical. The satellite is injected into a higher orbit with perigee at the release location; the orbital tether platform is injected into a lower orbit with apogee at the release location. The satellite enters a GTO trajectory and accomplishes the transfer in as little as 5-16 hours, where the lower number applies to a single-stage and the higher number to a twostage system. The platform then reboosts to its operational altitude using electric thrusters. The system thus achieves transfer times comparable to a chemical upper stage with the efficiencies of electric propulsion.

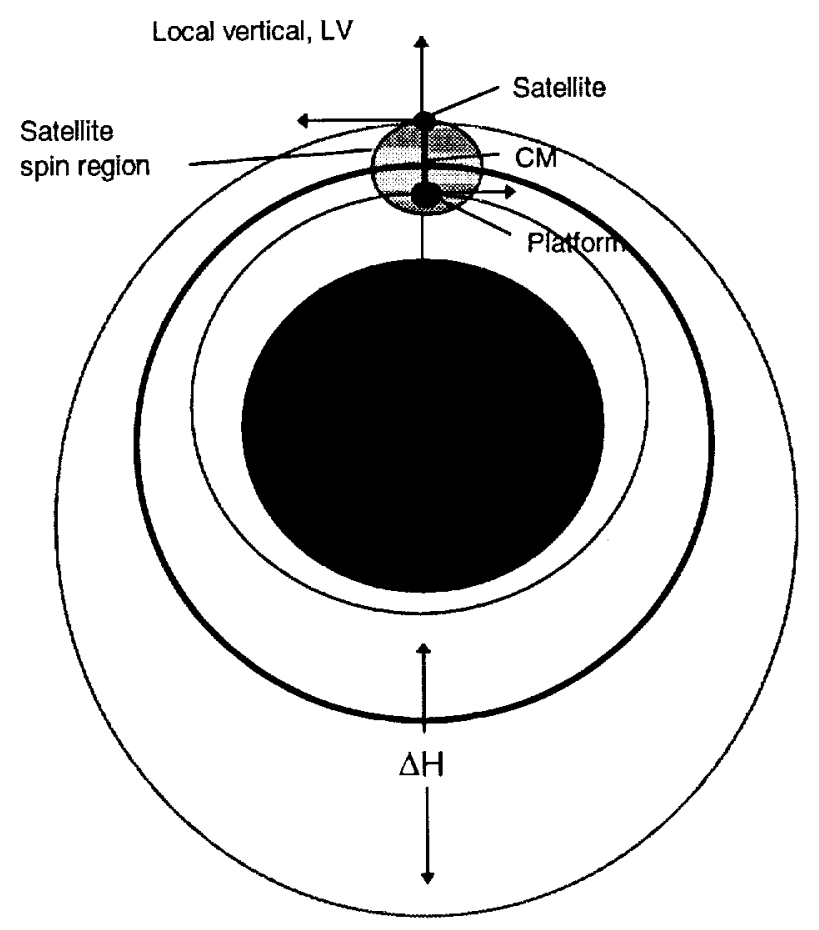

Fig. 6. Orbits of the launch vehicle and satellite after release in the 'spinning' tether boost scenario.

As an example, the length of an idealized single-stage system for transferring a $1,000 \mathrm{~kg}$ payload from LEO to a higher orbit is illustrated in Figure 7. The maximum acceleration is limited to $10 \mathrm{~g}$. An artist concept for the spinning momentum exchange tether transportation system is seen in Figure 8. 


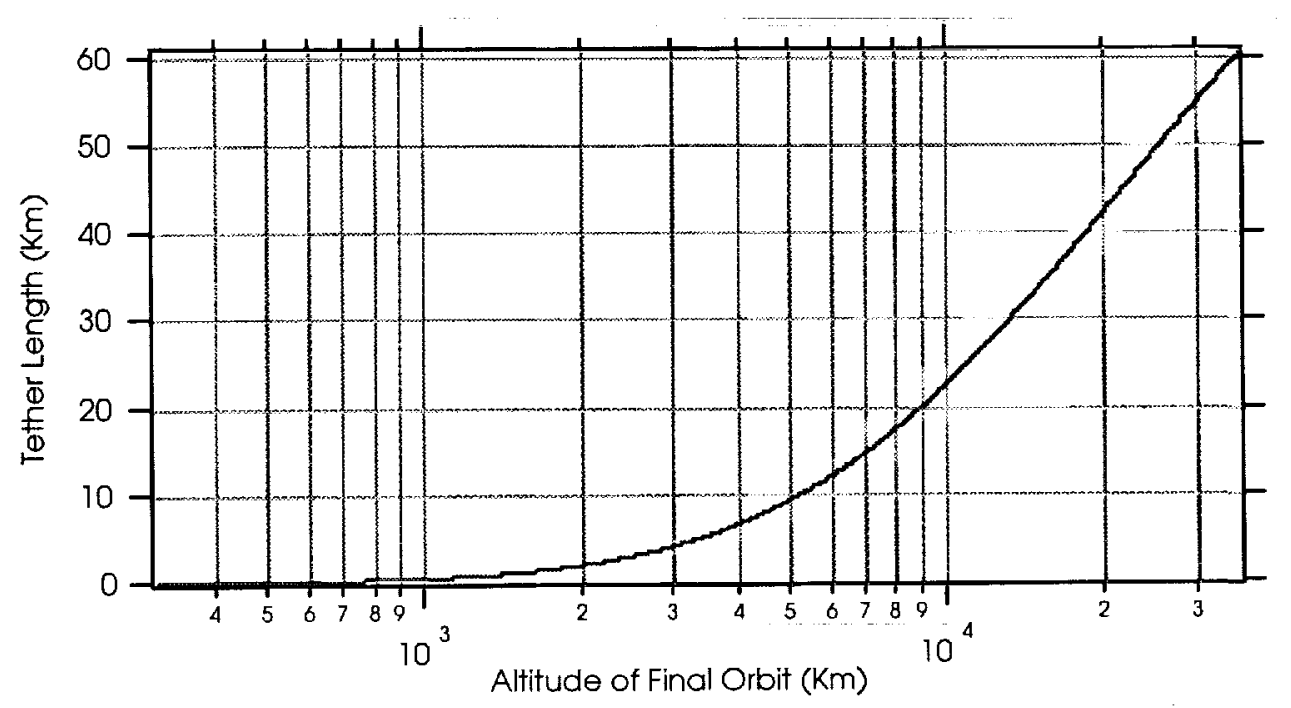

Fig. 7. Dimensions of a single-stage LEO tether transfer system.

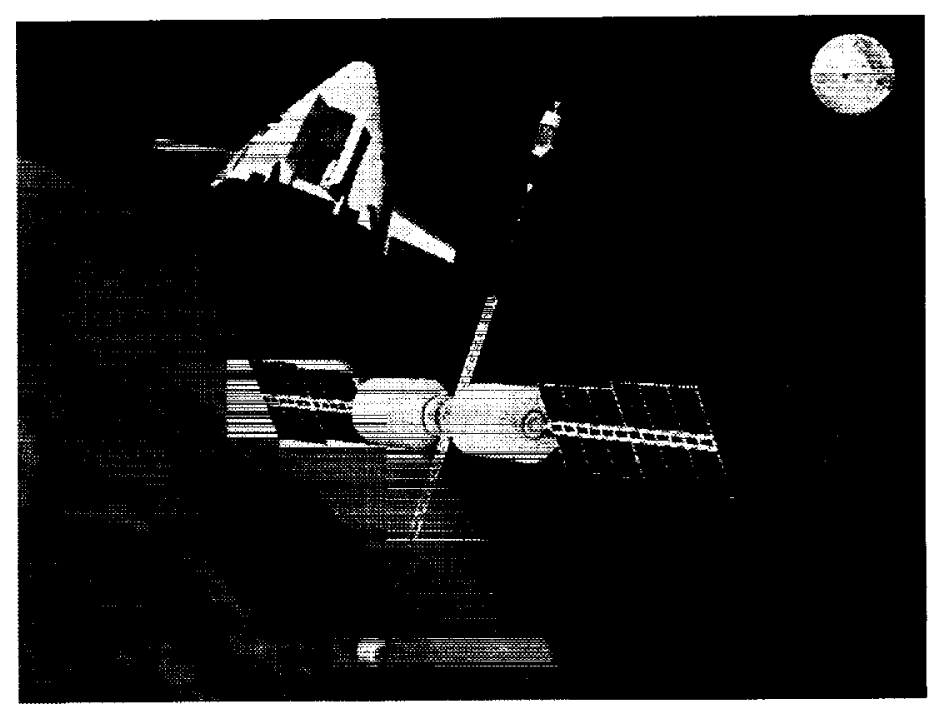

Fig. 8. Artist concept of a LEO-to-GTO tether transportation facility.

\section{TETHERS SUPPORTING SPACE SCIENCE}

Tether technology has matured to the point where it can now be applied to support scientific endeavors that do not center on the physics of the tether itself. Many of these proposed applications involve the scientific study of the Earth's upper atmosphere.

\section{An Upper Atmospheric Tether Mission}

Understanding the plasma and atmosphere around the Earth in the lower altitude regions of the mesosphere, lower thermosphere and ionosphere is important in research of the global electric system. An upper atmosphere tether mission has been proposed that would collect much needed data to further our knowledge of these regions. The proposed Shuttle mission would lower a tethered probe into certain regions of the Earth's atmosphere, collecting data over several days. A concept study recently defined an engineering system that would achieve the scientific objectives of the mission. 
The proposed tethered satellite mission offers the opportunity to make measurements required to unravel many of the complexities involved in ion-neutral coupling in the region between $200 \mathrm{~km}$ and $130 \mathrm{~km}$. Though of limited duration, a shuttle-based tethered satellite mission would provide the unique global access to a region of space required for significant understanding of the spatial scales of importance. The mission capitalizes on existing positive experiences from the tethered satellite program in a timely manner and represents an application of the human space flight program that can greatly contribute to our understanding of the Earth's environment.

Mission Scenario. The baseline scenario calls for a 6-day mission with the instrumented endmass deployed from the Orbiter for 2-days at each of the following altitudes: $170 \mathrm{~km}, 150 \mathrm{~km}$ and $130 \mathrm{~km}$. Two approaches, of varying complexity, are being considered.

Option 1: Deploy Only. The Orbiter enters a $220 \mathrm{~km}$ circular orbit at a $57^{\circ}$ inclination. On the first day the tethered endmass will be deployed downward $50 \mathrm{~km}$ to $170 \mathrm{~km}$ altitude and remain there for two days. On day three, an additional $20 \mathrm{~km}$ will be deployed, lowering the endmass to an altitude of $150 \mathrm{~km}$ for two days. On day five, the final $20 \mathrm{~km}$ of tether will be deployed, lowering the endmass to its final $130 \mathrm{~km}$ altitude where it will remain for two days. The Orbiter altitude will be maintained by use of its Primary Reaction Control System (PRCS). On day seven, the tether will be cut after which the endmass begins a reentry course.

Option 2: Deploy/Retrieve. The Orbiter enters a $280 \mathrm{~km}$ circular orbit at a $57^{\circ}$ inclination. On the first day the tethered endmass will be deployed downward $110 \mathrm{~km}$ to $170 \mathrm{~km}$ altitude. As the orbit of the Shuttle/tether system decays, tether is retrieved $(\sim 15 \mathrm{~km})$ to maintain the endmass at a constant $170 \mathrm{~km}$ altitude. On day three, the Orbiter enter a $265 \mathrm{~km}$ altitude orbit and an additional of $150 \mathrm{~km}$. Again, more tether is retrieved $(\sim 25 \mathrm{~km})$ to maintain the endmass at $150 \mathrm{~km}$ as the system altitude drops over the next two days. On day five, the Orbiter enters a $240 \mathrm{~km}$ orbit and deploys the final $20 \mathrm{~km}$ of tether, thus lowering the endmass to its final $130 \mathrm{~km}$ altitude where it will remain for two days. The Orbiter altitude will now be maintained by use of its Primary Reaction Control System (PRCS). On day seven, the tether will be cut after which the endmass begins a reentry course.

\section{Multipoint Science Using Tethers}

Multiple tethered satellites can be used to significantly advance our understanding of ionosphere thermosphere - magnetosphere coupling processes. The controlled vertical separation provided by a tethered configuration allows vertical gradients to be diagnosed while simultaneously sampling along a nominally horizontal satellite track. Since many of the coupling processes to be studied are highly dependent on the underlying vertical gradients in the background, the tethered satellite represents the only away to achieve this information on a global basis. Some of the parameters would only otherwise be available from sounding rockets and incoherent scatter radar and others, like the F-region neutral wind, can only be obtained with the required resolution from in-situ sampling.

An effort lead by The University of Texas at Dallas to conceptually define a mission to provide a tethered observation platform comprising three satellites in an elliptical orbit sampling the ionosphere and upper atmosphere in the altitude range from $140 \mathrm{~km}$ to $3000 \mathrm{~km}$ was selected for funding by NASA's Office of Space Science in 1997.

The mission concept assumes two satellites connected to a central spacecraft by up to 10 kilometers of long duration tether material. Each sub-satellite deploys itself from the central spacecraft by means of 
deployer system derived from the successful Small Expendable Deployer System (SEDS). Two smaller satellite platforms would be deployed upward and downward respectively from the central satellite. These two satellites would contain identical instrumentation

\section{SUMMARY}

Tether technology has advanced significantly since its inception over 30 years ago. The recent successes of the SEDS system show that tethers are ready to move from experiment and demonstration to application. One of the most promising applications for tethers is in the area of space propulsion and transportation. The use of electrodynamic tethers for reusable upper stages will soon be demonstrated with the ProSEDS mission and the technology is being considered for the use on the International Space Station. Momentum exchange tethers offer the highest payoff for transfer of payloads between LEO and GEO, GTO and lunar space. In addition, the many benefits of their use in support of space science has been recognized for many years. Missions to study the Earth's upper atmosphere are but two of many that have been proposed for study.

\section{REFERENCES}

Bekey, I., Tethering a new technique for payload deployment, Aerospace America, pp. 36-40, March (1997).

Colombo, G., Study of certain launching techniques using long tethers, NASA-CR-164060 (1981).

Rupp, C.C. and C. Carrington, MSFC Small Expendable Deployer System mission data, NASA Technical Memorandum (1993).

Sanmartin, J. R., M. Martinez-Sanchez and E. Ahedo, Bare wire anodes for electrodynamic tethers, J. of Propulsion and Power, 9(3), 353-360 (1993). 